\title{
Lecciones de Cuba el control y prevención de la pandemia por Covid-19 desde el papel de la Atención Primaria en Salud
}

\author{
Román Rafael Vega Romero, Diana Carolina Ruiz Mendoza, Valentina Martufi
}

\section{RESUMEN}

Revista da Rede APS 2021

Este relato de experiencia presenta como se organizó la respuesta a la pandemia causada por Covid-19 en Cuba. Se exponen algunas características generales del país y de su sistema de salud, el cual se caracteriza por ser un sistema público, universal, de acceso gratuito, basado en la APS. Además, se describen las estrategias implementadas para enfrentar la pandemia, entre las que se encuentran medidas de apoyo social a los más vulnerables, actividades comunitarias, acciones de vigilancia en salud pública, búsqueda activa de casos y contactos, atención a sintomáticos respiratorios, reorganización de la atención en los policlínicos, acompañamiento domiciliario. Se concluye que los resultados de la lucha contra la pandemia por Covid19 en Cuba son prometedores, se ha conseguido reducir la contagiosidad y la letalidad, sin descuidar la atención de las necesidades ordinarias de salud de la población.

Palabras-clave: Atención Primaria de Salud; COVID-19; Cuba.

\section{ABSTRACT}

This paper relates how the response to the Covid-19 pandemic was organized in Cuba. An overview is presented of the country and its health system, which is characterized as a public, free Access, universal system, centred on primary health care. In addition, the strategies implemented to face the pandemic are described, including social support measures for the most vulnerable, community activities, public health surveillance, active search for cases and contacts, care for respiratory patients, reorganization of care in polyclinics, and home monitoring. The results of the fight against the Covid-19 pandemic in Cuba are promising: it has been possible to reduce contagiousness and lethality, without neglecting the routine health needs of the population.

Keywords: Primary Health Care; COVID-19; Cuba.

Publicada em: 01/04/2021

DOI:10.14295/aps.v3i1.157

Román Rafael Vega Romero

(Pontificia Universidad Javeriana, Bogotá, Colômbia)

Diana Carolina Ruiz Mendonza (Instituto de Saúde Coletiva, Universidade Federal de Bahia, Salvador, Bahia, Brasil)

Valentina Martufi (Instituto de Saúde Coletiva, Universidade Federal de Bahia, Salvador, Bahia, Brasil)

Correspondência para:

Diana Ruiz

dcarorm@hotmail.com 


\section{INTRODUCCIÓN}

La pandemia causada por Covid-19 se ha diseminado de manera muy rápida y ha tomado por sorpresa a los distintos países. Las características políticas, económicas, sociales y sanitarias de cada nación han determinado diferentes afectaciones y respuestas en cada lugar.

Aunque en muchos países las acciones para el enfrentamiento a la pandemia se han enfocado en los cuidados especializados hospitalarios y en las acciones de vigilancia epidemiológica, la base para una respuesta adecuada a una emergencia como la actual es la Atención Primaria a la Salud (APS) con perspectiva comunitaria. Los sistemas de salud basados en la APS tienen la fortaleza de ser cercanos a la población, lo que puede contribuir con las acciones de vigilancia, la atención de casos probables de Covid-19, realizar referencia y contrareferencia a servicios especializados y continuar respondiendo a las necesidades rutinarias de las comunidades (Dunlop, 2020, Medina et al., 2020).

En esa perspectiva, la experiencia de enfrentamiento a la pandemia implementada en Cuba resulta relevante porque en ese país sus ciudadanos cuentan con un sistema de salud público de acceso universal, basado en un fuerte componente de vigilancia en salud pública, integrado a la APS y articulado con el sistema hospitalario.

Por tanto, en este artículo pretendemos describir brevemente la forma como se ha organizado la respuesta a la pandemia causada por Covid-19 en Cuba. Esto con la finalidad de sintetizar brevemente algunas lecciones aprendidas sobre como un sistema de salud aprovecha las capacidades de la APS para la prevención y control de la infección causada por el SARS-COV-2.

Este documento se construye a partir de la revisión de fuentes de información cubanas como Cubadebate, Granma, Prensa Latina, Juventud Rebelde, Bohemia, el apoyo de la Embajada de Cuba en Colombia, y de algunos artículos publicados.

\section{CUBA Y SU SISTEMA DE}

\section{SALUD}

La República de Cuba, es un Estado socialista de derecho y justicia social, democrático, independiente y soberano. Su forma principal de propiedad económica es la propiedad de todo el pueblo sobre los medios fundamentales de producción. Su sistema económico es de dirección planificada, la cual regula y controla el mercado "en función de los intereses de la sociedad" (CUBA, 2019, p.3)

La salud pública es un derecho de todas las personas, siendo el Estado responsable de garantizar "el acceso, la gratuidad y la calidad de los servicios de atención, protección y recuperación". Con este fin, el Estado ha creado un sistema de salud de acceso universal en todos los niveles -sustentado en los valores de solidaridad, equidad y derecho a la salud-, con énfasis en servicios de prevención y educación, y con participación de la sociedad y las familias. (CUBA, 2019, p.5)

El sistema está basado en un fuerte componente de vigilancia en salud pública integrado a la APS y en estrecha conexión con la red hospitalaria. El Programa de Trabajo del Médico y la Enfermera de Familia, - que es un modelo de APS centrado en la persona, la familia y la comunidad, con proyección territorial e intersectorial de salud -, es la base y puerta de entrada al sistema de salud (MORALES, 2018).

El modelo incluye un Equipo Básico de Salud (EBS) integrado por un médico y una enfermera de familia - que trabajan en un consultorio de familia de barrio y son responsables por 1500 personas. Cada EBS organiza su trabajo a través del modelo de planificación de acciones en salud. El médico de familia prioriza las actividades de consulta y realiza dos visitas en terreno por semana. Las actividades básicas de los EBS son la dispensarización - evaluación e intervención planificada y programada de personas y familias con base en el análisis de su situación de salud (individual, familiar y comunitaria), su registro, clasificación y seguimiento, atención domiciliaria y atención a 
personas en estado terminal. (GONZALEZ, 2018).

En cada Área de Salud (Grupo Básico de Trabajo - GBT) operan 15 a 20 EBS, atendiendo 20-30 mil personas, articulados con un Policlínico comunitario que cuenta con varias especialidades y servicios (pediatría, ginecología, obstetricia, medicina interna, psicología, optometría, estomatología, medicina natural, entre otras) y un hospital de referencia. A través de esa red integrada y con la coordinación de las acciones dentro de la red y con la población, mediante el uso de herramientas tecnológicas se garantiza la continuidad de la atención. (GONZALEZ, 2018)

Las acciones de vigilancia en salud pública forman parte integral de la estructura y de los procesos locales de la APS en los EBS y en los policlínicos comunitarios. A través de ellos tradicionalmente se realizan acciones de detección activa y pasiva de casos, diagnóstico, aislamiento, tratamiento temprano, rastreo $\mathrm{y}$ seguimiento de contactos, educación y comunicación para lograr comportamientos protectores, fomento de la participación comunitaria y la acción intersectorial en las actividades de promoción de la salud, prevención y control de la enfermedad. (VERDASQUERA, 2020)

El talento humano también caracteriza la APS en Cuba, la formación en medicina está orientada a la APS y la especialidad de Medicina General Integral se enfoca en formar médicos de familia con competencias centradas en atención individual, familiar, comunitaria y del ambiente. (VELA-VALDÉZ, 2018)

\section{CUBA Y LA PANDEMIA DE COVID-19}

\section{CARACTERÍSTICAS DE LA CIRCULACIÓN DE LA INFECCIÓN EN EL PAÍs}

Después de seis meses de la pandemia, cuyos tres primeros casos se confirmaron el 11 de marzo del 2020 y el primer fallecido el 17 del mismo mes, Cuba tenía 4726 casos confirmados -representando una tasa de prevalencia de Covid-19 por 100 mil habitantes de 41.5. Entre ellos, 4040 (85.5\%) se recuperaron, 108 (0.02\%) fallecieron, y $17(0.004 \%)$ estaban en estado crítico o grave (MINSAP, 2020).

De los 4726 pacientes diagnosticados con la enfermedad, $89,6 \%$ fueron contactos de casos confirmados, y $2775 \quad(58,7 \%)$ han sido asintomáticos. Hubo 488 casos en menores de 20 años, mientras el $81 \%$ de las muertes se produce entre mayores de 60 años, y el $38,7 \%$ entre mayores de 80 años. El día con más casos nuevos confirmados fue el 30 de abril (70) pero éstos han venido disminuyendo progresivamente con algunos días sin casos y otros con sólo un dígito de casos nuevos confirmados (MINSAP, 2020).

La distribución del virus fue predominante entre tres de las diez provincias más pobladas de Cuba -La Habana con 2819, Villa Clara con 270 y Matanzas con 273 casos-, en la provincia de Artemisa se registraron 356 casos, a pesar de estar entre las seis provincias menores del país. En cada una de las otras provincias se detectaron menos de 120 casos (CUBADEBATE, 2020).

\section{RESPUESTA DEL ESTADO Y DE LA SOCIEDAD A LA PANDEMIA}

\section{ACCIONES GENERALES}

A pesar del bloqueo económico, comercial y financiero impuesto por el gobierno de Estados Unidos, que ha impedido a Cuba comprar medicamentos, equipamientos e insumos de salud en el exterior, y del llamado de ese país a otros del mundo a rechazar la ayuda médica cubana, el gobierno de Cuba viene desarrollando una importante campaña de lucha contra el Covid-19 en su país y en varios países del mundo.

Las actividades de prevención y control de la pandemia incluyen reorganización del trabajo de las instituciones, regulación del comportamiento de las personas, vigilancia 
activa en salud pública, atención médica oportuna, ayuda a los grupos vulnerables, y participación de las comunidades y de las organizaciones de masas, entre otros. (VEGA, 2020)

Al principio de la pandemia, se organizaron audiencias sanitarias en las cuadras, centros de trabajo y de estudio para explicar la situación actual a la población, socializar las propuestas de respuesta del gobierno, y con la ayuda del personal de APS, definir cómo enfrentar la propagación del virus (FALCON ET AL, 2020).

Los medios de comunicación y redes sociales han contribuido en la divulgación de informaciones sobre las medidas de aislamiento, distanciamiento social, desinfección e higiene. En radio, televisión y otros medios informativos cubanos, son realizadas conferencias diarias de prensa del MINSAP, Mesas Redondas, teleclases y otros programas, que han posibilitado informar a la población sobre el curso de la enfermedad y las acciones para combatirla, con seguimiento en tiempo real de la situación en el país y en el mundo. (RAMON, 2020)

\section{ACCIONES DE APOYO SOCIAL Y PARA EL CUMPLIMIENTO DE LAS MEDIDAS DE AISLAMIENTO Y DISTANCIAMIENTO SOCIAL}

Conjuntamente el partido comunista, el gobierno y las organizaciones de masas toman las decisiones sobre las medidas de aislamiento y distanciamiento social. Entre las primeras medidas tomadas estuvo la suspensión de viajes turísticos aéreos, marítimos y terrestres, y el regreso de los turistas a sus países, con todas las medidas de prevención y control. El confinamiento de la población fue adoptado posteriormente. Sólo se permite la salida de una persona por familia para conseguir alimentos, asistir a servicios esenciales o para trabajar.

La población tiene garantizadas las condiciones para el cumplimiento de las medidas de aislamiento social. Los núcleos corrientes de personas vulnerables - concepto que no está asociado a ingresos insuficientes - se ampliaron con otros grupos, como adultos mayores que viven solos, personas en discapacidad, madres solteras con hijos menores, grupos en alto riesgo como enfermos crónicos que reciben el Servicio de Atención a la Familia (SAF), embarazadas, entre otros. En estos grupos se tienen contabilizadas 606.945 personas que son asistidas de diversas maneras.

El Ministerio de la Seguridad Social, a través de trabajadores sociales y personas reubicadas laboralmente, apoya a los vulnerables, las personas con ingresos insuficientes, en condiciones corrientes (aproximadamente 112.000 personas) que no están en condiciones de trabajar, ni tienen apoyo familiar, para que no tengan que salir de casa. Realizan actividades como trámites personales, entrega de ayudas monetarias, servicio de alimentación organizado en la comunidad, entrega de alimentos controlados, pago de pensiones y prestaciones de la seguridad social.

El trabajo voluntario, solidario y coordinado de los Comités de Defensa de la Revolución, y de la Federación de Mujeres Cubanas hace énfasis en la atención y entrega de alimentos a grupos vulnerables, confección y entrega de tapabocas, limpieza e higienización de hogares de ancianos con ayuda de jóvenes activistas, fomento del compartir el cuidado doméstico, prevención de la violencia de género, apoyo a las familias de los trabajadores de la salud, apoyo a los EBS en la pesquisa de sintomáticos y contactos, y para el acercamiento a embarazadas en zonas de riesgo, traslado de alimentos a hogares maternos y centros de aislamiento y atención a niños sin amparo, prisiones y centros de aseguradas. (FALCON ET AL, 2020)

Igualmente, la Federación de Estudiantes Universitarios participa en labores de pesquisa en las comunidades con estudiantes de medicina, en la distribución de alimentos y medicamentos, higienización, limpieza y vigilancia de calles, preparación de desinfectantes, producción de alimentos, trabajo en laboratorios que procesan pruebas PCR, instruyen a la población en cómo usar el pesquisador virtual y promueven el distanciamiento social. 
Integrantes del Ministerio del Interior, de las Fuerzas Armadas Revolucionarias (FAR), glorias del deporte y trabajadores del Instituto Nacional de Deporte, Educación Física y Recreación contribuyen para evitar la violación de las medidas de aislamiento social y aglomeraciones, realizan acciones educativas sobre medidas de higiene, protección personal y desinfección.

Las consignas son: «el momento que se está viviendo reclama el esfuerzo de todos para cumplir con la principal misión: salvar vidas», «es la hora de cuidarnos de cubano a cubano, con distanciamiento social. La vida es lo más preciado y preservándola también hacemos Revolución». (GONALEZ, 2020)

\section{RESPUESTA DEL SISTEMA DE SALUD A LA PANDEMIA}

El sistema de respuesta sanitaria a la pandemia por COVID-19 está basado en la vigilancia en salud pública, el modelo de medicina familiar, la red hospitalaria integrada a la salud pública y a la atención primaria a través de los Policlínicos comunitarios.

\section{LA APS Y SU PAPEL EN LA PANDEMIA}

La APS participa en las labores de prevención y control de la pandemia por el SARS-CoV-2 a través de acciones de vigilancia en salud y atención de pacientes infectados desde un enfoque de manejo sindrómico de la infección respiratoria aguda. La búsqueda activa de personas con síntomas respiratorios y sus contactos se realiza mediante georeferenciamiento en los domicilios $y$ comunidades a través de los EBS y mediante aplicaciones virtuales.

Está siendo usada una aplicación tecnológica virtual de uso ciudadano para la autopesquisa de salud, que permite identificar en tiempo real casos sintomáticos y sospechosos y adoptar conductas de vigilancia en salud. Esos posibles casos son redireccionados a las Áreas de Salud para que enfermeras y médicos de familia corroboren la información presencialmente mediante visita a los domicilios (FALCON ET AL, 2020)

Además de esas acciones, los EBS también identifican población en condiciones de vulnerabilidad como los ancianos solos, los pacientes con enfermedades crónicas no transmisibles, entre otros (FALCON ET AL, 2020).

Los EBS definen si los pacientes sospechosos permanecen en observación en casa, son remitidos a sitios de aislamiento (si su estado clínico es leve o moderado-), o si se remiten a servicios especializados (enfermos graves o en estado crítico).

Desde los consultorios barriales, los médicos y enfermeras de familia, y desde las policlínicas comunitarios otros profesionales, incluidos los estudiantes de medicina y estomatología debidamente entrenados y protegidos, con apoyo de la comunidad, hacen vigilancia, búsqueda activa y seguimiento diario casa a casa de casos sospechosos y contactos estrechos. Este personal enseña en las comunidades, en los sitios de trabajo y en las escuelas, medidas de prevención e autocuidado: lavado de manos, uso de tapabocas (en cubanismo, nasobuco), desinfección de lugares y objetos con agua clorada. También, realizan cercos sanitarios con aislamiento intensificado de lugares con brotes de la infección como hospitales, hogares de ancianos, centros de protección social, barrios. (FALCON ET AL, 2020).

La atención de sintomáticos respiratorios en los Policlínicos inicia con el triage que es realizado por una enfermera en la entrada, cumpliendo las medidas de higiene del paciente (lavado de manos, desinfección) y de protección personal (uso de tapabocas); a seguir se derivan los pacientes sospechosos (que tuvieron contacto con un enfermo por covid-19 o proceden de un país contagioso) a un área diferenciada de enfermedades respiratorias donde son atendidos por un médico y una enfermera, personal que está disponible 24 horas. Desde allí el paciente puede ser remitido al nivel de complejidad correspondiente, según la severidad de la enfermedad y la nacionalidad de la persona. El lugar es desinfectado cada vez que 
un paciente lo abandona. Los pacientes asintomáticos son vigilados en su domicilio por el respectivo EBS que les toma la temperatura dos veces por día durante 14 días. Los contactos de pacientes sospechosos y confirmados se mantienen en centros de aislamiento designados.

Se prioriza la atención del personal de salud que está en la primera línea de combate con equipos de protección personal, descanso, alojamiento y alimentación en los hospitales y centros de aislamiento, y aseguramiento de las familias. Además, se realiza atención psicológica por teléfono a la población y se promueve la solidaridad y el compañerismo frente a las personas en aislamiento o cuarentena.

\section{LA SOLIDARIDAD}

\section{INTERNACIONALISTA Y LA INVESTIGACIÓN}

Cuba tiene alrededor de 2041 trabajadores de la salud entre médicos, enfermeras, epidemiólogos y asesores para contribuir a atender la pandemia por COVID-19 en más de 28 brigadas médicas del Contingente Internacional Henry Reeve en países como Italia, Argentina, Sur África, Nicaragua, Haití, Angola, y Venezuela, entre otros, a pesar de la oposición de los Estados Unidos y de la derecha de algunos de esos países, que han buscado difamar y descalificar al personal médico cubano.

Cuba también sobresale en investigación científica e innovación tecnológica aplicada a la pandemia. El Interferón Alfa-2B Recombinante, un medicamento antiviral que Cuba viene produciendo con China, ha sido usado en este país como uno de los medicamentos contra la Covid-19. Está en desarrollo el antiviral Caletra, y están usando otros medicamentos de producción nacional como la Biomodulina $T$, la Itolizumab, el CIGB 258 y el plasma de pacientes recuperados, entre otros. (FALCON ET AL, 2020a)

\section{CONSIDERACIONES FINALES}

Los resultados de la lucha contra la pandemia por Covid-19 en Cuba son prometedores. Las medidas de confinamiento y distanciamiento social se han cumplido en gran parte, debido a acciones de apoyo social y comunicación de informaciones confiables a través de distintos medios.

Dichas acciones junto con el sistema de salud cubano, universal, de acceso gratuito, basado en la APS, la medicina familiar y comunitaria, con mirada territorial e intersectorial y las estrategias de vigilancia en salud pública, han sido fundamentales para el enfrentamiento a la pandemia.

A través de esa organización, en Cuba se ha conseguido reducir la contagiosidad y letalidad de la pandemia mediante acciones de diagnóstico temprano de casos, identificación de sospechosos, pesquisa de contactos y asintomáticos, aislamiento, seguimiento y tratamiento, con la participación de las comunidades y sin descuidar la atención de las necesidades ordinarias de salud de la población. 


\section{BIBLIOGRAFÍA}

Cubadebate. Cuba reporta 42 nuevos casos de COVID-19, ningún fallecido y 110 altas médicas. 14 sept 2020. Disponible: http://www.cubadebate.cu/noticias/2020/09/14/cuba-reporta-42-nuevos-casos-decovid-19-ningun-fallecido-y-110-altas-medicas-video/\#.X1 Ae8hKg2w Consultado: 1 jun 2020

Cuba. Constitución (2019). Constitución de la República de Cuba. Disponible en: http://www.granma.cu/file/pdf/gaceta/Nueva\%20Constituci\%C3\%B3n\%20240\%20KB-1.pdf_Consultado 10 mar 2020

Dunlop C, et al. The coronavirus outbreak: The central role of primary care in emergency preparedness and response. BJGP Open, vol4, n.1. 2020 Disponible en: https://bjgpopen.org/content/4/1/bjgpopen20X101041_Consultado: 28 may 2020

Falcon $\mathrm{R}$, et al. Cuba no relaja medidas ni se confía, pese a escenario favorable en el manejo de la COVID19. Cubadebate. 29 may 2020. Disponible: http://www.cubadebate.cu/noticias/2020/05/29/cuba-norelaja-medidas-ni-se-confia-pese-a-escenario-favorable-en-el-manejo-de-la-covid-19video/\#.XtKMC55KhR1 Consultado: 1 jun 2020

Falcon R, et al. COVID-19 en Cuba: Actualización epidemiológica y novedades de la ciencia. Cubadebate. 5 May 2020a. Disponible en: http://www.cubadebate.cu/noticias/2020/05/05/covid-19-en-cubaactualizacion-epidemiologica-y-novedades-de-la-ciencia-video/\#.XslxGp5KiL8 Consultado: 1 jun 2020a

González LT, et al. El Programa del Médico y Enfermera de la Familia: desarrollo del modelo de atención médica en Cuba. Rev Panam Salud Publica. Vol.42, e31. 2018 https://doi.org/10.26633/RPSP.2018.31

González O. Destacamento se integra a la lucha contra expansión de la COVID-19. Granma. La Habana, 22 abr 2020. Disponible en: http://www.granma.cu/cuba-covid-19/2020-04-22/destacamento-se-integra-ala-lucha-contra-expansion-de-la-covid-19-22-04-2020-02-04-41. Consultado: 20 may 2020

Medina MG, et al. Atenção Primária à Saúde em tempos de Covid-19: O que fazer? Cad. Saúde Pública. vol.36, n.8. 2020 Disponível em: http://cadernos.ensp.fiocruz.br/csp/artigo/1140/atencao-primaria-asaude-em-tempos-de-covid-19-o-que-fazer. Consultado: 30 ago 2020

MINSAP. Ministerio de la Salud Pública. Parte de cierre del día 13 de septiembre a las 12 de la noche. Disponible en: https://salud.msp.gob.cu/parte-de-cierre-del-dia-13-de-septiembre-a-las-12-de-la-noche/ Consultado: 14 sep 2020

Morales Ojeda R, et al. Transformaciones en el sistema de salud en Cuba y estrategias actuales para su consolidación y sostenibilidad. Rev Panam Salud Publica. Vol42, e25. 2018. https://doi.org/10.26633/ RPSP.2018.25. Consultado: 20 may 2020

Ramón M. Radio y televisión cubanas informan y entretienen en tiempos de COVID-19. Cubadebate. 18 May del 2020. Disponible en: http://www.cubadebate.cu/noticias/2020/05/18/radio-y-televisioncubanas-informan-y-entretienen-en-tiempos-de-covid-19/\#.XslyPp5KiL8 Consultado: 1 jun 2020

Vega R. Covid-19: Lecciones de Cuba desde la Atención Primaria en salud. Voz Partido Comunista Colombiano. 2 jun 2020. Disponible en: http://www.pacocol.org/index.php/noticias/salud/11883-covid19-lecciones-de-cuba-desde-la-atencion-primaria-en-salud Consultado: 24 jul 2020

Vela-Valdés J, et al. Formación del capital humano para la salud en Cuba. Rev Panam Salud Publica. Vol42, e33. 2018. Disponible en: https://doi.org/10.26633/RPSP.2018.33. Consultado: 25 may 2020. 
Verdasquera Corcho D, et al. Capacidad de respuesta y desafíos del sistema de salud cubano frente a las enfermedades transmisibles. Rev Panam Salud Publica. Vol42, e30. Disponible en: https://doi.org/10.26633/RPSP.2018.30. Consultado: 25 may 2020 